\title{
3. Secret writings and attitudes - research questions
}

This research has two objectives in order to fill the gaps in the study of cryptography and secrecy. First, it aims to reconstruct the social milieus of the applicants and the reception of cryptography, not only in diplomacy (where ciphers were used in the largest quantity and in their most professional form), but also in science, religion, artisanal tradition, university context, espionage, medicine, and in the private lives of noblemen, engineers, and everyday people, where previous research had neglected its role. Second, it aims to integrate cryptography into the larger intellectual context of secrecy, in private, medical, scientific, religious, alchemical, magical, and political practices of secrecy, that is, in the context where it suigeneris belongs.

The number of research questions to be answered is extensive. What was the relationship between various practices of intentional secrecy and cryptography? On what occasions were secret writings used and what were the alternative tools available at the time?

What was the content of the hidden knowledge: politics, sexual secrets, or scientific knowledge? What type of information did the contemporaries want to be secret? The question is not only whether we can solve the ciphers, but also: Can we figure out which texts were seen as important enough to be enciphered? This is even more relevant when the given information does not seem today valuable enough to be hidden. It is worthwhile to compare in a given source the enciphered contents with and those that were not to better understand people's attitudes to secrecy.

The ways of knowledge transfer would be equally important to discover. How did techniques of cryptography spread in society, e.g. by way of printed texts, by manuscripts, or by personal transfer? Was the source of their knowledge the diplomatic practice or the manuals of such classic authors of cryptography as Johannes Trithemius, Athanasius Kircher, and Giambattista Della Porta? Which deciphering methods in the region were results of endogenous development, and which came from the Ottoman Empire? Was there any knowledge transfer between the considerably more developed Arabic tradition the slowly improving European cipher practices? If such transfer did take place, did this happen in the territory of Hungary, where the Islamic and European cultures confronted each other, and where double spies were familiar with both the Eastern and Western 
methods? If, on the other hand, such transfer did not take place, what was the reason for its lack?

How widespread were the encryption and decrypting methods outside the political sphere? Is there any correlation between the quality and sophistication of a cipher system being used and the social status, education, and the distance of the user from the practice of diplomacy?

How much trust was laid in these methods? What was the exact purpose for enciphering a text: to make it cryptic and hidden from the contemporaries, or something else? Why did certain diaries use ciphers that were easily solvable? How can we identify whether a specific cipher key was used for diplomatic missions or for the communication of private secrets?

How can the perception of risk that justified enciphering be reconstructed on the basis of the sources? What are the - often civilian - practitioners' attitudes towards the technology they used? How far could they make use of the techniques, how far did they realize the potential of the given methods? What complications took place because of misunderstood encryption? Which mistakes were typically made by users when applying ciphers, or more precisely, how might they have decreased the efficiency of their techniques? What measures were made to protect the secret of a specific key? How frequently did users change their encryption? To what extent were they aware that the key might be broken by their enemies? How developed and practical was the diplomatic practice of cryptography? Was encryption and code breaking carried out by the clerks or did the ruler himself felt obliged to spend precious time enciphering and deciphering secret reports?

The questions abound. To summarize the, one can use a paraphrase of Jacques Le Goff's famous words: 'what is common to the university student, the emperor's clerk, and the master of the mint as far as their attitudes to and practices of cryptography were concerned?

1 The histoire des mentalités operates at the level of the everyday automatisms of behavior. Its object is that which escapes historical individuals because it reveals the impersonal content of their thought: that which is common to Caesar and his most junior legionary, Saint Louis and the peasant on his lands, Christopher Columbus and any one of his sailors. The histoire des mentalités is to the history of ideas as the history of material culture is to economic history. Jacques le Goff and Pierre Nora, Constructing the Past: Essays in Historical Methodology (Cambridge: Cambridge UP, 1985), 169. 